\title{
SUFFICIENT CONDITIONS FOR A PROBLEM OF MAYER IN THE CALCULUS OF VARIATIONS*
}

\author{
BY \\ G. A. BLISS AND M. R. HESTENES
}

1. Introduction. The general problem of Mayer with variable end points as proposed by Bliss $(\mathrm{V}, \mathrm{p} .305) \dagger$ is that of finding in a class of arcs

$$
y_{i}=y_{i}(x) \quad\left(x_{1} \leqq x \leqq x_{2} ; i=1, \cdots, n\right)
$$

satisfying a system of differential equations and end conditions

$$
\begin{array}{lr}
\phi_{\alpha}\left(x, y, y^{\prime}\right)=0 & (\alpha=1, \cdots, m<n), \\
\psi_{\mu}\left[x_{1}, y\left(x_{1}\right), x_{2}, y\left(x_{2}\right)\right]=0 & (\mu=1, \cdots, p \leqq 2 n+1)
\end{array}
$$

one which minimizes a function of the form

$$
g\left[x_{1}, y\left(x_{1}\right), x_{2}, y\left(x_{2}\right)\right] .
$$

Bliss has shown that this problem is equivalent to a problem of Bolza (V, p. 306) in the sense that each can be transformed into one of the other type. For the problem of Bolza the function to be minimized is

$$
I=g\left[x_{1}, y\left(x_{1}\right), x_{2}, y\left(x_{2}\right)\right]+\int_{x_{1}}^{x_{2}} f\left(x, y, y^{\prime}\right) d x,
$$

and it is clear at once that the problem of Mayer is a problem of Bolza having $f \equiv 0$.

Sufficient conditions for the problem of Bolza have been established by Morse (XI, p. 528) and Bliss (XII, p. 271). However the hypotheses which they make, in particular that of normality on every sub-interval, imply that the function $f$ is not identically zero, and the sets of sufficient conditions established by them are therefore not applicable to the problem of Mayer without further modification. In view of this fact it is the purpose of the authors of the present paper to establish a set of sufficient conditions for the problem of Mayer with variable end points. This will be done in two parts, the first of which is the paper here presented, dealing only with the special case in which the number of end conditions $\psi_{\mu}=0$ is exactly $2 n+1$. By methods similar to those used by Bliss for the problem of Bolza (XII, pp. 261-274) the results obtained will be extended to the general case in a second paper by Hestenes.

\footnotetext{
* Presented to the Society, April 8, 1932; received by the editors June 9, 1932.

$\dagger$ Roman numerals in parentheses refer to the bibliography at the end of this paper.
} 
The problem considered here is an obvious generalization of the classical problem of Mayer and reduces to the latter when the expression to be minimized is the function $g=y_{1}\left(x_{2}\right)$ and the end conditions $\psi_{\mu}=0$ are the conditions

$$
\begin{aligned}
x_{1}-\alpha_{1}=y_{i}\left(x_{1}\right)-\beta_{i 1}=x_{2}-\alpha_{2} & =y_{j}\left(x_{2}\right)-\beta_{j 2}=0 \\
& (i=1, \cdots, n ; j=2, \cdots, n),
\end{aligned}
$$

the $\alpha$ 's and $\beta$ 's being constants. Sufficiency theorems for the classical problem have been established by Egorov (II, p. 376), Kneser (I, p. 250; VIII, p. 290), and Larew (VII, p. 65), who use in each case an $n$-dimensional field defined in the $(n+1)$-dimensional space of points $\left(x, y_{1}, \ldots, y_{n}\right)$ by an $(n-1)$ parameter family of extremals passing through a fixed point. Such a field does not seem to be applicable to the problem considered here, but one can use instead a field of $n+1$ dimensions defined by an $n$-parameter family of extremals in $\left(x, y_{1}, \cdots, y_{n}\right)$-space. The construction and use of such a field are important features of this paper. An $(n+1)$-dimensional field of this sort is applicable to the more special classical problem of Mayer also, and a fundamental sufficiency theorem for this case can be established in this way with greater ease and fewer restrictions than have hitherto been required.

2. Preliminary remarks. In the following pages it is assumed that the various indices have the following ranges unless otherwise explicitly specified:

$$
\begin{gathered}
i, k=1,2, \cdots, n ; \quad \alpha, \beta=1,2, \cdots, m<n ; \\
\rho, \sigma=1,2, \cdots, 2 n+1 ; \quad r=1,2, \cdots, n-1 ; \\
s=1,2, \cdots, 2 n-1 .
\end{gathered}
$$

The tensor analysis summation convention is used freely throughout. We make the following hypotheses concerning a particular arc $E_{12}$ whose minimizing properties are to be studied:

(a) The functions $y_{i}(x)$ defining $E_{12}$ are continuous on the interval $x_{1} x_{2}$, and this interval can be subdivided into a finite number of parts on each of which these functions have continuous derivatives.

(b) The functions $\phi_{\alpha}$ have continuous partial derivatives of the first three orders in a neighborhood $\Re$ of the values $\left(x, y, y^{\prime}\right)$ on $E_{12}$, and at each element $\left(x, y, y^{\prime}\right)$ in $\Re$ the matrix $\left\|\phi_{\alpha \nu_{k}^{\prime}}\right\|$ has rank $m$.

(c) The functions $g, \psi_{\rho}$ have continuous partial derivatives of the first two orders in a neighborhood of the end values $\left(x_{1}, y_{i 1}, x_{2}, y_{i 2}\right)$ of $E_{12}$ in which the determinant

$$
\left|\begin{array}{llll}
g_{x_{1}} & g_{y_{i 1}} & g_{x_{2}} & g_{y_{i 2}} \\
\psi_{\rho x_{1}} & \psi_{\rho y_{i 1}} & \psi_{\rho x_{2}} & \psi_{\rho y_{i 2}}
\end{array}\right|
$$

is different from zero. 
An admissible set $\left(x, y, y^{\prime}\right)$ is a set interior to $\Re$ and satisfying the equations $\phi_{\alpha}=0$. An arc (1:1) having the continuity properties described in (a) is called admissible if all of its elements $\left(x, y, y^{\prime}\right)$ are admissible. The definitions of equations of variation and of admissible variations used in the following pages are those of Bliss (V, p. 307; IX, p. 677). The problem of Mayer here proposed can now be more precisely stated as that of finding in the class of admissible arcs satisfying the end conditions $\psi_{\rho}=0$ one which minimizes the function $g$.

I. The FIRST NECESSARY CONDITION. For every minimizing arc $E_{12}$ for the problem of Mayer as here proposed there exist constants $c_{i}$ and a function $F=\lambda_{\alpha}(x) \phi_{\alpha}$ such that the equations

$$
F_{y_{i^{\prime}}}=\int_{x_{1}}^{x} F_{y_{i}} d x+c_{i}, \phi_{\alpha}=0
$$

are satisfied at each point of $E_{12}$. The multipliers $\lambda_{\alpha}(x)$ are continuous except possibly at the values of $x$ defining corners of $E_{12}$ and do not vanish simultaneously at any point of $E_{12}$.

To prove this theorem one needs only to combine the methods used by Bliss for the corresponding theorems in the problems of Mayer (V, p. 311) and Lagrange (IX, p. 683). It is also an immediate corollary of a theorem established by Morse and Myers for the problem of Bolza (X, p. 245).

THEOREM 2:1. If the functions $\lambda_{\alpha}(x)$ are a set of multipliers with which an admissible arc $E_{12}$ satisfies the equations (2:2), then for every set of admissible variations $\xi_{1}, \xi_{2}, \eta_{i}(x)$ along $E_{12}$ the functions $\eta_{i}(x)$ satisfy the equations

$$
\left.F_{y_{i^{\prime}}} \eta_{i}\right|_{x^{\prime}} ^{x^{\prime \prime}}=0
$$

for every interval $x^{\prime} x^{\prime \prime}$.

This result is readily provable by multiplying the equations of variation

$$
\phi_{\alpha y_{i}} \eta_{i}+\phi_{\alpha y_{i}{ }^{\prime}} \eta_{i}^{\prime}=0
$$

by the multipliers $\lambda_{\alpha}(x)$, adding, and applying the usual integration by parts with the help of equations $(2: 2)$.

An admissible arc $E_{12}$ is said to be normal relative to the end conditions $\psi_{\rho}=0$ if there exist for it $2 n+1$ sets of admissible variations $\xi_{1}^{\sigma}, \xi_{2}^{\sigma}, \eta_{i}^{\sigma}(x)$ such that the determinant $\left|\Psi_{\rho}\left(\xi^{\sigma}, \eta^{\sigma}\right)\right|$ is different from zero, where

$$
\Psi_{\rho}(\dot{\xi}, \eta)=\left(\psi_{\rho x_{1}}+y_{i 1}{ }^{\prime} \psi_{\rho y_{i 1}}\right) \xi_{1}+\psi_{\rho y_{i 1}} \eta_{i 1}+\left(\psi_{\rho x_{2}}+y_{i 2}{ }^{\prime} \psi_{\rho y_{i 2}}\right) \xi_{2}+\psi_{\rho y_{i 2}} \eta_{i 2}
$$


the functions $y_{i}, y_{i}^{\prime}$ occurring explicitly and in the derivatives of $\psi_{\rho}$ being those belonging to $E_{12}$. The arc $E_{12}$ is normal on the sub-interval $x^{\prime} x^{\prime \prime}$ if there exist for it $2 n-1$ sets of admissible variations $\xi_{1}^{s}, \xi_{2}^{s}, \eta_{i}^{s}(x)$ such that the matrix

$$
|| \begin{gathered}
\eta_{i}{ }^{8}\left(x^{\prime}\right) \\
\eta_{i}{ }^{(2}\left(x^{\prime \prime}\right)
\end{gathered}||
$$

has rank $2 n-1$. On account of the relation (2:3) this is the highest rank attainable for a matrix with columns of this sort belonging to an arc that satisfies the equations (2:2) with a set of multipliers $\lambda_{\alpha}(x)$. For convenience an arc that is normal relative to the end conditions $\psi_{\rho}=0$ will be designated simply as normal.

THEOREM 2:2. An admissible arc $E_{12}$ that satisfies the necessary condition $\mathrm{I}$ is normal if and only if there exist for it no set of multipliers $\lambda_{\alpha}(x)$, not vanishing simultaneously, with which it satisfies equations (2:2) and for which the determinant

$$
\left|\begin{array}{cccc}
0 & F_{y_{i^{\prime}}}\left(x_{1}\right) & 0 & -F_{y_{i^{\prime}}}\left(x_{2}\right) \\
\psi_{\rho x_{1}}+y_{i 1}^{\prime} \psi_{\rho y_{i 1}} & \psi_{\rho y_{i 1}} & \psi_{\rho x_{2}}+y_{i 2}^{\prime} \psi_{\rho y_{i 2}} & \psi_{\rho y_{i 2}}
\end{array}\right|
$$

vanishes on $E_{12}$. If $E_{12}$ is normal the constant $l_{0}$ defined below can be taken equal to 1 , and its multipliers $\lambda_{\alpha}(x)$ are then unique.

To prove the theorem we first notice that the arc $E_{12}$ is normal if and only if there exist for it no set of constants and multipliers $l_{0}, l_{\rho}, \lambda_{\alpha}(x)$ having $l_{0}=0$ but not vanishing simultaneously with which it satisfies the relations $(2: 2)$ and

$$
\begin{aligned}
l_{0}\left(g_{x_{1}}+y_{i 1}^{\prime} g_{v_{i 1}}\right)+l_{\rho}\left(\psi_{\rho x_{1}}+y_{i 1}^{\prime} \psi_{\rho y_{i 1}}\right) & =0, \\
l_{0} g_{y_{i 1}} & =l_{\rho} \psi_{\rho y_{i 1}} \\
l_{0}\left(g_{x_{2}}+y_{i 2}^{\prime} g_{y_{i 2}}\right)+l_{\rho}\left(\psi_{\rho x_{2}}+y_{i 2}{ }^{\prime} \psi_{\rho y_{i 2}}\right) & =0, \\
l_{0 g_{y_{i 2}}}+l_{\rho} \psi_{\rho y_{i 2}} & =-F_{y^{\prime}}\left(x_{2}\right) .
\end{aligned}
$$

This criterion for normality is readily established by the same methods as those used by Bliss for the case when $E_{12}$ is an extremal ( $\left.V, p .311\right)$. If for a set of multipliers $\lambda_{\alpha}(x)$ belonging to $E_{12}$ the determinant (2:5) vanishes, then there is a set $l_{0}, l_{\rho}, c \lambda_{\alpha}(x)$ having $l_{0}=0$ and satisfying the equations $(2: 6)$. Hence $E_{12}$ could not be normal. On the other hand if the determinant (2:5) is different from zero for every set of multipliers $\lambda_{\alpha}(x)$ with which $E_{12}$ satisfies equations $(2: 2)$, then there can be no set $l_{0}, l_{\rho}, \lambda_{\alpha}(x)$ with $l_{0}=0$ satisfying the equations $(2: 6)$. Consequently in this case $E_{12}$ is normal. The last statement in the theorem is readily established by the methods used by Bliss for the case when $E_{12}$ is an extręmal $(\mathrm{V}$, p. 311$)$. 
THEOREM 2:3. If an admissible arc $E_{12}$ is normal on $x^{\prime} x^{\prime \prime}$ and satisfies the equations (2:2) with a set of multipliers $\lambda_{\alpha}(x)$, then these multipliers are unique on the interval $x^{\prime} x^{\prime \prime}$ except for a constant factor.

This is a result of the relation $(2: 3)$ which implies that the constants $F_{y_{i^{\prime}}}\left(x^{\prime}\right), F_{y_{i^{\prime}}}\left(x^{\prime \prime}\right)$ are unique except for a constant factor since it is possible to select a matrix $(2: 4)$ having rank $2 n-1$ on $x^{\prime} x^{\prime \prime}$. The multipliers belonging to $E_{12}$ on the interval $x^{\prime} x^{\prime \prime}$ are then also unique except for a constant factor since they are completely determined when the set of values $F_{y_{i^{\prime}}}\left(x^{\prime}\right)$ is specified (IX, p. 680).

3. The family of extremals. An extremal is an admissible arc with a set of multipliers not vanishing simultaneously

$$
y_{i}=y_{i}(x), \quad \lambda_{\alpha}=\lambda_{\alpha}(x) \quad\left(x_{1} \leqq x \leqq x_{2}\right)
$$

which have continuous derivatives $y_{i}^{\prime}(x), y_{i}^{\prime \prime}(x), \lambda_{\alpha}^{\prime}(x)$ and satisfy the EulerLagrange equations

$$
(d / d x) F_{y_{i}^{\prime}}-F_{y_{i}}=0, \quad \phi_{\alpha}=0 .
$$

Such an extremal is non-singular if the determinant

$$
R=\left|\begin{array}{lc}
F_{y_{i^{\prime}} y_{k^{\prime}}} & \phi_{\beta y_{i^{\prime}}} \\
\phi_{\alpha y_{k^{\prime}}} & 0
\end{array}\right|
$$

is different from zero along it. Along a non-singular extremal $E_{12}$ the equations

$$
F_{y^{\prime}}\left(x, y, y^{\prime}, \lambda\right)=z_{i}, \quad \phi_{\alpha}\left(x, y, y^{\prime}\right)=0
$$

can be solved for the variables $y_{i}^{\prime}, \lambda_{\alpha}$ in a neighborhood of the values $(x, y, z)$ on the arc $E_{12}$. The solution has the form

$$
y_{i}^{\prime}=P_{i}(x, y, z), \quad \lambda_{\alpha}=\Lambda_{\alpha}(x, y, z),
$$

and has continuous partial derivatives of the first two orders since the first members of equations $(3: 2)$ have such derivatives. The system of equations $(3: 1)$ is now equivalent to the system

$$
d y_{i} / d x=P_{i}(x, y, z), \quad d z_{i} / d x=F_{y_{i}}[x, y, P(x, y, z), \Lambda(x, y, z)] .
$$

The functions $F, P_{i}, \Lambda_{\alpha}$ satisfy the homogeneity relations

$$
\begin{array}{rlr}
F\left(x, y, y^{\prime}, k \lambda\right) & =k F\left(x, y, y^{\prime}, \lambda\right), \\
P_{i}(x, y, k z) & =P_{i}(x, y, z), \\
\Lambda_{\alpha}(x, y, k z) & =k \Lambda_{\alpha}(x, y, z)
\end{array}
$$

The first of these relations is a consequence of the definition of $F$. The last two follow from the fact that the two sets 


$$
\begin{aligned}
& {[x, y, k z, P(x, y, z), k \Lambda(x, y, z)],} \\
& {[x, y, k z, P(x, y, k z), \Lambda(x, y, k z)]}
\end{aligned}
$$

satisfy equations (3:2) and must be identical since the solutions $P, \Lambda$ of these equations are unique when $x, y, z$ are given.

Through every element $\left(x_{0}, y_{0}, z_{0}\right)$ in a neighborhood of the set of values $(x, y, z)$ on the extremal $E_{12}$ there passes a unique solution

$$
y_{i}=y_{i}\left(x, x_{0}, y_{0}, z_{0}\right), \quad z_{i}=z_{i}\left(x, x_{0}, y_{0}, z_{0}\right)
$$

of equations (3:4) for which the functions $y_{i}, y_{i x}, z_{i}, z_{i x}$ have continuous partial derivatives of the first two orders since the second members of equations $(3: 4)$ have such derivatives. The functions $y_{i}\left(x, x_{0}, y_{0}, z_{0}\right), k z_{i}\left(x, x_{0}, y_{0}, z_{0}\right)$ are solutions of equations ( $3: 4)$, on account of the homogeneity properties (3:5), and have the initial values $(x, y, z)=\left(x_{0}, y_{0}, k z_{0}\right)$. Since the solutions with these initial values are unique it follows that

$$
\begin{aligned}
& y_{i}\left(x, x_{0}, y_{0}, k z_{0}\right)=y_{i}\left(x, x_{0}, y_{0}, z_{0}\right), \\
& z_{i}\left(x, x_{0}, y_{0}, k z_{0}\right)=k z_{i}\left(x, x_{0}, y_{0}, z_{0}\right) .
\end{aligned}
$$

Since each curve $(3: 6)$ has an initial set at $x=x_{10}$ we lose none of them if we replace $x_{0}$ by the fixed value $x_{10}$. Furthermore not all the constants $z_{i 0}$ are zero at the initial element of $E_{12}$. We may therefore renumber the solutions $(3: 6)$ so that $z_{n 0}$ is different from zero. On account of the homogeneity relations (3:7) it follows that the initial elements $\left(x_{10}, y_{0}, z_{0}\right),\left(x_{10}, y_{0}, k z_{0}\right)$ determine the same curves $y_{i}=y_{i}\left(x, x_{10}, y_{0}, z_{0}\right)$. Hence we lose none of these curves if we assign to $z_{n 0}$ the fixed value of $z_{n}$ belonging to $E_{12}$ at the point 1 . Let us for convenience rename the constants $y_{10}, y_{20}, \cdots, y_{n 0}, z_{10}, \cdots, z_{n-1,0}$ and call them $c_{1}, c_{2}, \cdots, c_{2 n-1}$ respectively. The family $(3: 6)$ then takes the form

$$
y_{i}=y_{i}(x, c), \quad z_{i}=z_{i}(x, c)
$$

The equations

$$
c_{i}=y_{i}\left(x_{10}, c\right), \quad c_{n+r}=z_{r}\left(x_{10}, c\right), \quad z_{n 0}=z_{n}\left(x_{10}, c\right)
$$

express the fact that the solutions (3:8) pass through the initial element

$$
\left(x, y_{1}, \cdots, y_{n}, z_{1}, \cdots, z_{n-1}, z_{n}\right)=\left(x_{10}, c_{1}, \cdots, c_{n}, c_{n+1}, \cdots, c_{2 n-1}, z_{n 0}\right)
$$

and from them we find by differentiation that the determinant

$$
\left|\begin{array}{cc}
y_{i c_{s}} & 0 \\
z_{i c_{s}} & z_{i}
\end{array}\right|
$$

takes the value $z_{n 0}$ at $x=x_{10}$. When we substitute the functions $(3: 8)$ in 
equations (3:3) a set of functions $\lambda_{\alpha}(x, c)$ is determined, and we have the final result:

THEOREM 3:1. Every non-singular extremal arc $E_{12}$ is a member of a $(2 n-1)$ parameter family of extremals

$$
y_{i}=y_{i}(x, c), \quad \lambda_{\alpha}=\lambda_{\alpha}(x, c) \quad\left(x_{1} \leqq x \leqq x_{2}\right)
$$

for special values $\left(x_{1}, x_{2}, c\right)=\left(x_{10}, x_{20}, c_{0}\right)$. The functions $y_{i}, y_{i x}, z_{i}, z_{i x}, \lambda_{\alpha}$ have continuous first and second partial derivatives in a neighborhood of the values $(x, c)$ defining $E_{12}$, and for the special values $\left(x_{10}, c_{0}\right)$ the determinant (3:9) is different from zero.

4. The second variation for a normal extremal. Consider a normal extremal arc $E_{12}$ with ends satisfying the conditions $\psi_{\rho}=0$. Let $\xi_{1}, \xi_{2}, \eta_{i}(x)$ be a set of admissible variations along $E_{12}$ satisfying the equations $\Psi_{p}(\xi, \eta)=0$. It can be shown that there is a one-parameter family of admissible arcs

$$
y_{i}=y_{i}(x, b), \quad x_{1}(b) \leqq x \leqq x_{2}(b),
$$

satisfying the end conditions $\psi_{\rho}=0$, containing $E_{12}$ for $b=0$, and having $\xi_{1}, \xi_{2}, \eta_{i}(x)$ as its variations along $E_{12}$ (IX, p. 695). The functions $x_{1}(b), x_{2}(b)$, $y_{i}(x, b), y_{i b}(x, b)$ are continuous in a neighborhood of the values $(x, b)$ defining $E_{12}$, and their derivatives $x_{1 b}, x_{1 b b}, x_{2 b}, x_{2 b b}, y_{i x}, y_{i x b b}, y_{i b b}$ have the same property except possibly at the values of $x$ defining the corners of the arc $\eta_{i}=\eta_{i}(x)$ $\left(x_{1} \leqq x \leqq x_{2}\right)$ in $x \eta$-space.

When the equations

$$
\begin{aligned}
g(b) & =g\left[x_{1}(b), y\left(x_{1}(b), b\right), x_{2}(b), y\left(x_{2}(b), b\right)\right], \\
0 & =\psi_{\rho}\left[x_{1}(b), y\left(x_{1}(b), b\right), x_{2}(b), y\left(x_{2}(b), b\right)\right], \\
0 & =\phi_{\alpha}\left[x, y(x, b), y^{\prime}(x, b)\right]
\end{aligned}
$$

are multiplied by constants and multipliers $l_{0}, l_{\rho}, \lambda_{\alpha}(x)$, where $l_{0}, l_{\rho}$ are to be determined later and the functions $\lambda_{\alpha}(x)$ are the multipliers belonging to $E_{12}$, it is found by suitable additions that

$$
\begin{aligned}
l_{0} g(b) & =G\left[x_{1}(b), y\left(x_{1}(b), b\right), x_{2}(b), y\left(x_{2}(b), b\right)\right], \\
0 & =F\left[x, y(x, b), y^{\prime}(x, b), \lambda_{\alpha}(x)\right],
\end{aligned}
$$

where $G=l_{0} g+l_{\rho} \psi_{\rho}$. By differentiating these equations for $b$ it follows further that

$$
\begin{aligned}
\log ^{\prime}(b)= & \left(G_{x_{1}}+y_{i 1}^{\prime} G_{y_{i 1}}\right) x_{1 b}+G_{y_{i 1}} y_{i b}\left(x_{1}\right) \\
& +\left(G_{x_{2}}+y_{i 2}^{\prime} G_{y_{i b}}\right) x_{2 b}+G_{y_{i 2}} y_{i b}\left(x_{2}\right), \\
0= & F_{y_{i} y_{i b}}+F_{y_{i}^{\prime}} y_{i b}^{\prime},
\end{aligned}
$$


and a second differentiation gives for $b=0$

$$
\begin{aligned}
l_{0} g^{\prime \prime}(0)= & \left(G_{x_{1}}+y_{i 1}^{\prime} G_{y_{i 1}}\right) x_{1 b b}+\left.G_{y_{i_{1}}} y_{i b b}\left(x_{1}\right)\right|^{b=0} \\
& +\left(G_{x_{2}}+y_{i 2}^{\prime} G_{y_{i 2}}\right) x_{2 b b}+\left.G_{y_{i 2}} y_{i b b}\left(x_{2}\right)\right|^{b=0} \\
& +Q\left[\xi_{1}, \eta\left(x_{1}\right), \xi_{2}, \eta\left(x_{2}\right)\right], \\
0= & F_{y_{i} y_{i b b}}+\left.F_{y_{i}} y_{i b b}\right|^{b=0}+2 \omega\left(x, \eta, \eta^{\prime}\right),
\end{aligned}
$$

where $Q$ is a quadratic form in the variations $\xi_{1}, \eta_{i}\left(x_{1}\right), \xi_{2}, \eta_{i}\left(x_{2}\right)$ of the family (4:1) along $E_{12}$ and

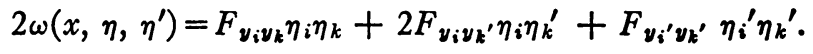

When equation (4:3) is integrated from $x_{1}$ to $x_{2}$, it is found with the help of the Euler-Lagrange equations (3:1) that

$$
0=\left.F_{y_{i}^{\prime}} y_{i b b}\right|_{x_{1}} ^{x_{2}}+\int_{x_{1}}^{x_{2}} 2 \omega\left(x, \eta, \eta^{\prime}\right) d x .
$$

From the hypothesis (c) of $\S 2$, and since $E_{12}$ is normal, we can determine the constants $l_{0}, l_{\rho}$ to satisfy equations $(2: 6)$ with $l_{0}=1$. Hence by adding equations (4:2) and (4:5) it follows that the second variation $I_{2}$ along $E_{12}$ can be expressed in the form

$$
I_{2}=g^{\prime \prime}(0)=Q\left[\xi_{1}, \eta\left(x_{1}\right), \xi_{2}, \eta\left(x_{2}\right)\right]+\int_{x_{1}}^{x_{2}} 2 \omega\left(x, \eta, \eta^{\prime}\right) d x,
$$

and this expression must be $\geqq 0$ for every set of admissible variations $\xi_{1}, \xi_{2}$, $\eta_{i}(x)$ along $E_{12}$ satisfying the conditions $\Psi_{\rho}(\xi, \eta)=0$.

Since $E_{12}$ is normal the relation (2:3) and Theorem 2:2 imply that every set of admissible variations $\xi_{1}, \xi_{2}, \eta_{i}(x)$ along $E_{12}$ satisfying the conditions $\Psi_{\rho}=0$ also satisfies the equations $\xi_{1}=\eta_{i}\left(x_{1}\right)=\xi_{2}=\eta_{i}\left(x_{2}\right)=0$. Hence in the expression (4:6) the value of the quadratic form $Q$ is always zero, and we have the following theorem:

THEOREM 4:1. Along a normal extremal arc $E_{12}$ with ends satisfying the conditions $\psi_{\rho}=0$ the second variation is always expressible in the form

$$
I_{2}=\int_{x_{1}}^{x_{2}} 2 \omega\left(x, \eta, \eta^{\prime}\right) d x
$$

for all admissible variations $\xi_{1}, \xi_{2}, \eta_{i}(x)$ satisfying the equations $\Psi_{\rho}=0$, where $2 \omega$ is the quadratic form (4:4). If $g\left(E_{12}\right)$ is to be a minimum for the problem of Mayer as here proposed, then this second variation must be $\geqq 0$ for every set of admissible variations $\eta_{i}(x)$ satisfying the relations

$$
n_{i}\left(x_{1}\right)=n_{i}\left(\mathrm{x}_{2}\right)=0 \text {. }
$$


Since the functions $\eta_{i}(x)$ satisfy the differential equations of variation

$$
\Phi_{\alpha}\left(x, \eta, \eta^{\prime}\right)=\phi_{\alpha \nu_{i} \eta_{i}}+\phi_{\alpha \nu_{i}} \eta_{i}^{\prime}=0
$$

it is clear that the properties of the second variation suggest a minimum problem which is a problem of Lagrange (cf. VI, p. 16), namely, that of minimizing $I_{2}$ in the class of arcs

$$
\eta_{i}=\eta_{i}(x)
$$$$
\left(x_{1} \leqq x \leqq x_{2}\right)
$$

satisfying equations $(4: 8)$ and passing through the fixed points $\left(x_{1}, 0\right)$, $\left(x_{2}, 0\right)$ in $x \eta$-space as indicated by equations $(4: 7)$. One readily verifies that this problem is abnormal since, as was seen in §2, the rank of the matrix $(2: 4)$ cannot exceed $2 n-1$ on $E_{12}$. However, by a suitable modification of the end conditions the problem can be made normal. For this purpose we replace the condition that the arc (4:9) passes through the fixed points $\left(x_{1}, 0\right),\left(x_{2}, 0\right)$ in $x \eta$-space by the conditions

$$
x_{1}-\alpha_{1}=\eta_{i}\left(x_{1}\right)=x_{2}-\alpha_{2}=\eta_{l}\left(x_{2}\right)=0 \quad(l=1, \cdots, n ; l \neq p),
$$

where $p$ is chosen so that $F_{y^{\prime}}\left(x_{2}\right) \neq 0$. The two sets of end conditions are equivalent since the relation $(2: 3)$ implies that $\eta_{p}\left(x_{2}\right)=0$ whenever the conditions $(4: 10)$ are satisfied.

To prove that the new accessory problem just described is normal we use the fact that since $E_{12}$ is normal there is a determinant of the form $\left|\Psi_{\rho}\left(\xi^{\sigma}, \eta^{\sigma}\right)\right|$ which is different from zero on $E_{12}$. The matrix of this determinant is the product of two matrices, the first of which is formed by deleting the first row of the matrix (2:5) and has rank $2 n+1$, and the second of which is a matrix having $2 n+1$ columns of the form

$$
\xi_{1}^{\sigma}, \eta_{i}^{\sigma}\left(x_{1}\right), \xi_{2}^{\sigma}, \eta_{i}^{\sigma}\left(x_{2}\right) .
$$

This second matrix must also have rank $2 n+1$ if the original determinant is to be different from zero, and the determinant formed from this second matrix by leaving out the row of elements $\eta_{p}{ }^{\sigma}\left(x_{2}\right)$ must be different from zero, as one readily sees with the help of the relation $(2: 3)$. This last determinant is however one of the form whose non-vanishing insures the normality of the accessory problem with end conditions $(4: 10)$.

The Euler-Lagrange equations for the $x \eta$-problem are the equations

$$
(d / d x) \Omega_{\eta_{i^{\prime}}}-\Omega_{\eta_{i}}=0, \quad \Phi_{\alpha}\left(x, \eta, \eta^{\prime}\right)=0,
$$

where $\Omega\left(x, \eta, \eta^{\prime}, \mu\right)=\mu_{0} \omega+\mu_{\alpha} \Phi_{\alpha}$. These equations are known as the accessory equations for the original Mayer problem. 
THEOREM 4:2. If the functions $\mu_{0}=1, \mu_{\alpha}(x)$ are a set of multipliers with which an admissible arc (4:9) for the $x \eta$-problem satisfies equations (4:12), then every set of functions $\rho_{0}=1, \rho_{\alpha}(x)$ having this property is of the form $\rho_{0}=1$, $\rho_{\alpha}(x)=\mu_{\alpha}(x)+k \lambda_{\alpha}(x)$, where the functions $\lambda_{\alpha}(x)$ are the multipliers for $E_{12}$ and $k$ is an arbitrary constant.

This follows because if $\rho_{0}=1, \rho_{\alpha}(x)$ are a second set of multipliers for the arc (4:9), then the differences $\rho_{\alpha}(x)-\mu_{\alpha}(x)$ must be multipliers for the original problem and hence be of the form $\rho_{\alpha}(x)-\mu_{\alpha}(x)=k \lambda_{\alpha}(x)$, since $E_{12}$ is normal. This proves the theorem (cf. VI, p. 19).

An admissible arc (4:9) having associated with it a set of multipliers $\mu_{0}$, $\mu_{\alpha}(x)$ with which it satisfies equations $(4: 12)$ will also satisfy the transversality condition for the accessory problem just described if it satisfies the relation $\Omega_{\eta^{\prime}}\left(x_{2}\right)=0$ (IX, p. 693). Since $E_{12}$ is normal and $F_{y^{\prime}}\left(x_{2}\right) \neq 0$ it follows that a solution $\eta_{i}(x), \rho_{0}=1, \rho_{\alpha}=\mu_{\alpha}(x)+k \lambda_{\alpha}(x)$ of equations $(4: 12)$ satisfies the transversality condition $\Omega_{n p^{\prime}}\left(x_{2}\right)=0$ for a suitably selected value of the constant $k$.

Let us now assume that $E_{12}$ is also non-singular. Then the determinant $R$ is different from zero along $E_{12}$, and the equations

$$
\Omega_{\eta_{i^{\prime}}}\left(x, \eta, \eta^{\prime}, \mu\right)=\zeta_{i}, \quad \Phi_{\alpha}\left(x, \eta, \eta^{\prime}\right)=0
$$

with $\mu_{0}=1$ can be solved for the variables $\eta_{i}^{\prime}, \mu_{\alpha}$. The solution has the form

$$
\eta_{i}^{\prime}=H_{i}(x, \eta, \zeta), \quad \mu_{\alpha}=M_{\alpha}(x, \eta, \zeta),
$$

and the accessory equations (4:12) with $\mu_{0}=1$ are now equivalent to the equations

$$
\begin{aligned}
& d \eta_{i} / d x=H_{i}(x, \eta, \zeta), \\
& d \zeta_{i} / d x=\Omega_{\eta_{i}}[x, \eta, H(x, \eta, \zeta), M(x, \eta, \zeta)],
\end{aligned}
$$

which are linear and homogeneous in the variables $\eta_{i}, \zeta_{i}$. They have the solution $\eta_{i} \equiv 0, \zeta_{i}=z_{i}(x)$, where $z_{i}(x)$ are the values of the derivatives $F_{y_{i}}$ along $E_{12}$, since the corresponding values $\eta_{i} \equiv 0, \mu_{\alpha}=\lambda_{\alpha}$ reduce the first equations $(4: 12)$ to the Euler-Lagrange equations $(3: 1)$. It is known that for equations (4:13) a set of $2 n-1$ solutions $u_{i s}, v_{i s}$, whose determinant

$$
\left|\begin{array}{ll}
u_{i s} & 0 \\
v_{i s} & z_{i}
\end{array}\right|
$$

is different from zero for one value of $x$, has that determinant different from zero for all values of $x$. Furthermore every solution $\left(\eta_{i}, \zeta_{i}\right)$ of equations $(4: 13)$ is expressible in the form

$$
\eta_{i}=c_{s} u_{i s}, \quad \zeta_{i}=c_{s} v_{i s}+k z_{i},
$$


where $c_{s}, k$ are constants (IV, pp. 153-4). One readily verifies that the columns of the determinant (3:9) are a set of solutions of equations (4:13) like those in the columns of (4:14) (IX, p. 726).

As an immediate consequence of the relation (4:15) it follows that there is one and only one solution $\left(\eta_{i}, \zeta_{i}\right)$ of equations $(4: 12)$ taking prescribed values $\eta_{i 0}, \zeta_{i 0}$ at a given value $x_{0}$. In particular the only solution taking the values $\eta_{i 0}=\zeta_{i 0}=0$ at $x=x_{0}$ is the solution $\eta_{i} \equiv \zeta_{i} \equiv 0$. Furthermore, since $E_{12}$ is normal the only solution having $\eta_{i} \equiv 0$ on $x_{1} x_{2}$ is the solution $\eta_{i} \equiv 0, \zeta_{i}=$ $k z_{i}(x)$. The same is true on a sub-interval $x^{\prime} x^{\prime \prime}$ provided $E_{12}$ is normal on this sub-interval.

5. The necessary condition of Mayer. A value $x_{3} \neq x_{1}$ is said to define a point 3 conjugate to 1 on $E_{12}$ if there exists a solution $\eta_{i}=u_{i}(x), \mu_{0}=1$, $\mu_{\alpha}=\rho_{\alpha}(x)$ of equations (4:12) whose functions $u_{i}(x)$ satisfy the relations $u_{i}\left(x_{1}\right)=u_{i}\left(x_{3}\right)=0$ but are not all identically zero on $x_{1} x_{3}$.

IV. THE NECESSARY CONDITION OF MAYER. Let $E_{12}$ be a non-singular normal extremal arc, normal on every pair of sub-intervals $x_{1} x_{3}$ and $x_{3} x_{2}$. If $E_{12}$ is a minimizing arc for the problem of Mayer as here proposed, then between 1 and 2 on $E_{12}$ there can be no points 3 conjugate to 1 .

If there were a solution $\eta_{i}=u_{i}(x), \mu_{0}=1, \mu_{\alpha}=\rho_{\alpha}(x)$ of equations (4:12) whose functions $u_{i}(x)$ vanish at $x_{1}$ and $x_{3}$ but are not all identically zero on $x_{1} x_{3}$, then for the functions $\eta_{i}(x), \mu_{0}, \mu_{\alpha}(x)$ defined by the equations

$$
\begin{array}{llll}
\eta_{i}(x) \equiv u_{i}(x), & \mu_{0}=1, & \mu_{\alpha}(x) \equiv \rho_{\alpha}(x) \text { on } x_{1} x_{3}, \\
\eta_{i}(x) \equiv 0, & \mu_{0}=1, & \mu_{\alpha}(x) \equiv 0 & \text { on } x_{3} x_{2}
\end{array}
$$

the second variation $I_{2}$ would take the value zero (IX, p. 726). It follows that the arc

$$
\eta_{i}=\eta_{i}(x) \quad\left(x_{1} \leqq x \leqq x_{2}\right)
$$

would be a minimizing arc for the $x \eta$-problem since $E_{12}$ is to be a solution of the original problem. Hence there would be associated with the $\operatorname{arc}(5: 2)$ a function $\Omega=\omega+\mu_{\alpha} \Phi_{\alpha}$ with which it would satisfy the accessory equations (4:12), the transversality condition $\Omega_{\eta_{p^{\prime}}}\left(x_{2}\right)=0$, and the condition that the derivatives $\Omega_{\eta_{i^{\prime}}}(x)$ are continuous on the interval $x_{1} x_{2}$. As was seen above the most general multipliers possible for the functions $\eta_{i}(x)$ would have the forms $\mu_{0}=1, \mu_{\alpha}=\rho_{\alpha}(x)+c \lambda_{\alpha}(x)$ on the interval $x_{1} x_{3}$ and $\mu_{0}=1, \mu_{\alpha}=d \lambda_{\alpha}(x)$ on the interval $x_{3} x_{2}$. On account of the transversality condition $\Omega_{\eta_{p^{\prime}}}\left(x_{2}\right)=0$ it is found that $d=0$ since $F_{v_{p^{\prime}}}\left(x_{2}\right) \neq 0$. Hence at $x=x_{3}$ the corner condition would require 


$$
\Omega_{\eta^{\prime}}\left(x_{3}-0\right)=\omega_{\eta i^{\prime}}\left(x, u, u^{\prime}\right)+\left.\left(\rho_{\alpha}+c \lambda_{\alpha}\right) \phi_{\alpha y_{1^{\prime}}}\right|^{x_{3}}=0 .
$$

It follows that there would exist for the arc $(5: 2)$ a set of multipliers $\mu_{0}=1$, $\mu_{\alpha}=\rho_{\alpha}(x)+c \lambda_{\alpha}(x)$ such that at $x=x_{3}$ the functions $\zeta_{i}=\Omega_{\eta_{i^{\prime}}}\left(x, u, u^{r}, \rho+c \lambda\right)$ vanish as well as $\eta_{i}=u_{i}$. Hence the functions $\eta_{i}(x), \zeta_{i}(x)$ would all vanish identically on $x_{1} x_{3}$ which is not the case, and the theorem is therefore established (cf. VI, p. 18).

6. The determination of conjugate points. Consider a non-singular, normal extremal arc $E_{12}$ that is normal on every sub-interval $x_{1} x_{3}$.

THEOREM 6:1. Let $u_{i s}, v_{\text {is }}$ be $2 n-1$ solutions of equations (4:13) whose determinant (4:14) is different from zero at $x=x_{1}$. A value $x_{3} \neq x_{1}$ determines $a$ point 3 conjugate to 1 on $E_{12}$ if and only if the matrix

$$
|| \begin{aligned}
& u_{i s}\left(x_{3}\right) \\
& u_{i s}\left(x_{1}\right)
\end{aligned}||
$$

has rank $<2 n-1$.

This theorem is a simple extension of a theorem given by Larew and can be proved by the same methods (VI, p. 20).

If now we select $2 n-1$ solutions $u_{i s}, v_{i s}$ of equations (4:13), as in Theorem $6: 1$, and such that at $x=x_{1}$ the functions $u_{i s}(x)$ have the values

$$
u_{i r}\left(x_{1}\right)=0, \quad u_{i, n-1+k}\left(x_{1}\right)=\delta_{i k} \quad\left(\delta_{i i}=1, \delta_{i k}=0 \text { for } i \neq k\right),
$$

then it is clear that the matrix $(6: 1)$ for this set has rank $2 n-1$ if and only if the matrix $\left\|u_{i r}\left(x_{3}\right)\right\|$ has rank $n-1$. With this in mind we can prove the following theorem:

THEOREM 6:2. Let $u_{i k}, v_{i k}$ be $n$ solutions of equations $(4: 13)$ which at $x=x_{1}$ satisfy the relations

$$
\begin{array}{ll}
u_{i r}\left(x_{1}\right)=0, & \left|v_{i r}\left(x_{1}\right) z_{i}\left(x_{1}\right)\right| \neq 0, \\
u_{i n}\left(x_{1}\right)=z_{i}\left(x_{1}\right), & v_{i n}\left(x_{1}\right)=0 .
\end{array}
$$

$A$ value $x_{3} \neq x_{1}$ determines a point 3 conjugate to 1 on $E_{12}$ if and only if $D\left(x_{3}\right)=0$, where $D(x)=\left|u_{i k}(x)\right|$.

The theorem follows at once from our previous considerations if we show that $D\left(x_{3}\right)$ vanishes if and only if the matrix $\left\|u_{i r}\left(x_{3}\right)\right\|$ has rank $<n-1$. If now $D\left(x_{3}\right)=0$, then there exist constants $a_{k}$, not all zero, such that $u_{i k}\left(x_{3}\right) a_{k}$ $=0$. On account of the relation $(2: 3)$ for the functions $\eta_{i}(x)=u_{i k}(x) a_{k}$ and the values of $u_{i k}$ at $x=x_{1}$ it follows that 


$$
0=z_{i}\left(x_{3}\right) u_{i k}\left(x_{3}\right) a_{k}=z_{i}\left(x_{1}\right) u_{i k}\left(x_{1}\right) a_{k}=z_{i}\left(x_{1}\right) z_{i}\left(x_{1}\right) a_{n} .
$$

Hence $a_{n}=0$, and the matrix $\left\|u_{i r}\left(x_{3}\right)\right\|$ has rank $<n-1$. The converse is immediate, and the theorem is established.

7. Mayer fields and a fundamental sufficiency theorem. The importance of the introduction of the notion of an $(n+1)$-dimensional field in the space of points $\left(x, y_{1}, \cdots, y_{n}\right)$ for the problems of Mayer will be seen from the following considerations.

Definition of A MAYer FIELD. A Mayer field for the problem considered in this paper is a region $\mathfrak{F}$ in $x y$-space containing only interior points and having associated with it a set of functions $p_{i}(x, y), \lambda_{\alpha}(x, y)$ with the following properties:

(a) they have continuous first partial derivatives in $\mathfrak{F}$;

(b) the sets $[x, y, p(x, y)]$ defined by the points $(x, y)$ in $F$ are all admissible;

(c) the integral

$$
I^{*}=\int\left\{F(x, y, p, \lambda) d x+\left(d y_{i}-p_{i} d x\right) F_{y_{i^{\prime}}}(x, y, p, \lambda)\right\}
$$

formed with these functions is independent of the path in $\mathfrak{F}$.

This definition of a field is precisely the one given by Bliss for the problem of Lagrange except for the form of the function $F(\mathrm{IX}, \mathrm{p} .730)$. It should be noted that for the problem of Mayer here discussed the function $F(x, y, p, \lambda)$ vanishes identically in $\mathfrak{F}$, which is not in general true for the problems of Lagrange. Bliss has shown that the solutions $y_{i}(x)$ of the equations $d y_{i} / d x$ $=p_{i}(x, y)$ are extremals with multipliers $\lambda_{\alpha}(x, y(x))$, called extremals of the field. It is clear that the value of $I^{*}$ is zero along every extremal of the field.

THEOREM 7:1. If $E_{12}$ is a normal extremal arc of a field $\mathfrak{F}$ with ends satisfying the conditions $\psi_{\rho}=0$, then there is a neighborhood $N$ of the ends of $E_{12}$ in $\left(x_{1} y_{1} x_{2} y_{2}\right)$-space such that for every admissible arc $C_{34}$ in $\mathfrak{F}$ with ends in $N$ satisfying the conditions $\psi_{\rho}=0$ the formula

$$
g\left(C_{34}\right)-g\left(E_{12}\right)=\left(1 / \lambda_{0}\right) \int_{x_{3}}^{x_{4}} E\left[x, y, p(x, y), \lambda(x, y), y^{\prime}\right] d x
$$

holds, where $\lambda_{0}$ is a suitably chosen positive constant,

$$
E\left(x, y, p, \lambda, y^{\prime}\right)=F\left(x, y, y^{\prime}, \lambda\right)-F(x, y, p, \lambda)-\left(y_{i}^{\prime}-p_{i}\right) F_{y^{\prime}}(x, y, p, \lambda),
$$

and the arguments $y_{i}(x), y_{i}^{\prime}(x)$ occurring in the integrand are those belonging to $C_{34}$. 
As a first step in the proof consider the equations

$$
g\left(x_{1}, y_{1}, x_{2}, y_{2}\right)=g, \quad \psi_{\rho}\left(x_{1}, y_{1}, x_{2}, y_{2}\right)=0 .
$$

By hypothesis they are satisfied by the set $\left[x_{1}, y_{1}, x_{2}, y_{2}, g\left(E_{12}\right)\right]$ belonging to $E_{12}$. Since the determinant $(2: 1)$ is different from zero these equations have solutions of the form

$$
x_{1}=x_{1}(g), \quad y_{i 1}=y_{i 1}(g), \quad x_{2}=x_{2}(g), \quad y_{i 2}=y_{i 2}(g)
$$

which have continuous second derivatives in a neighborhood of the value $g=g\left(E_{12}\right)$. Furthermore, in a sufficiently small neighborhood $N$ of the ends of $E_{12}$ the only solutions are those defined by equations $(7: 2)$. These equations define two arcs $A, B$ through the ends of $E_{12}$.

The equations

$$
\begin{array}{lr}
l_{0} g_{x_{1}}+l_{\rho} \psi_{\rho x_{1}}= & -\left.p_{i} F_{y_{i^{\prime}}}(x, y, p, \lambda)\right|^{1}, \\
l_{0} g_{y_{i 1}}+l_{\rho} \psi_{\rho y_{i 1}}= & \left.F_{y_{i^{\prime}}}(x, y, p, \lambda)\right|^{1}, \\
l_{0} g_{x_{2}}+l_{\rho} \psi_{\rho x_{2}}= & \left.p_{i} F_{y_{i^{\prime}}}(x, y, p, \lambda)\right|^{2}, \\
l_{0 g_{y_{i 2}}+l_{\rho} \psi_{\rho y_{i 2}}}=- & -\left.F_{y_{i^{\prime}}}(x, y, p, \lambda)\right|^{2},
\end{array}
$$

where the variables $x_{1}, y_{i 1}, x_{2}, y_{i 2}$ are replaced by the right members of equations $(7: 2)$, determine continuous functions $l_{0}(g), l_{\rho}(g)$. When they are multiplied by the differentials $d x_{1}, d y_{i 1}, d x_{2}, d y_{i 2}$ belonging to the $\operatorname{arcs} A, B$ and added, it is found that

$$
l_{0} d g=-\left.F_{y_{i^{\prime}}}\left(d y_{i}-p_{i} d x\right)\right|_{1} ^{2} .
$$

In order to compare the values of $g$ for the $\operatorname{arcs} E_{12}$ and $C_{34}$ this last equation may be integrated from $g=g\left(E_{12}\right)$ to $g=g\left(C_{34}\right)$. By then applying the first law of the mean to the left member, an equation of the form

$$
\lambda_{0}\left[g\left(C_{34}\right)-g\left(E_{12}\right)\right]=I^{*}\left(A_{13}\right)-I^{*}\left(B_{24}\right)
$$

is obtained, where $\lambda_{0}$ is a suitably selected mean value of the function $l_{0}(g)$ on $E_{12}$. Since $E_{12}$ is normal we may suppose $l_{0}=1$ on $E_{12}$, according to the agreement made in $\$ 2$. Consequently the neighborhood $N$ can be chosen so small that $l_{0}(g)>0$ and hence $\lambda_{0}>0$ in $N$. Furthermore, since $I^{*}$ is independent of the path in $\mathfrak{F}$ it is clear that

$$
I^{*}\left(A_{13}\right)-I^{*}\left(B_{24}\right)=I^{*}\left(E_{12}\right)-I^{*}\left(C_{34}\right)=-I^{*}\left(C_{34}\right),
$$

the last equality being valid since $I^{*}$ vanishes identically along the extremal $E_{12}$ of the field. The theorem now follows at once from equations $(7: 4)$ and $(7: 5)$ since, as is easily seen, the value of $-I^{*}\left(C_{34}\right) / \lambda_{0}$ is equal to the value of the second member of equation $(7: 1)$. 
It is now possible to prove the following important theorem:

Theorem 7:2. A FUNDAMENTAL SUFFICIENCY THEOREM. Let a normal extremal arc $E_{12}$ be an extremal of a field $\mathfrak{F}$. Suppose that the ends of $E_{12}$ satisfy the conditions $\psi_{\rho}=0$ and that there is a neighborhood $N$ of these ends in $\left(x_{1} y_{1} x_{2} y_{2}\right)$ space such that no other extremal of the field has ends in $N$ satisfying the equations $\psi_{p}=0$. If at each point of $\mathfrak{F}$ the condition

$$
E\left[x, y, p(x, y), \lambda(x, y), y^{\prime}\right]>0
$$

holds for every admissible set $\left(x, y, y^{\prime}\right) \neq(x, y, p)$, then the neighborhood $N$ can be so restricted that the inequality $g\left(C_{34}\right)>g\left(E_{12}\right)$ is true for every admissible arc $C_{34}$ in $\mathfrak{F}$ with ends in $N$ satisfying the conditions $\psi_{\rho}=0$ and not identical with $E_{12}$.

To prove this, restrict $N$ so as to be effective as in Theorem $7: 1$. It follows at once from Theorem 7:1 that the inequality $g\left(C_{34}\right) \geqq g\left(E_{12}\right)$ is necessarily satisfied by every admissible arc $C_{34}$ in $\mathfrak{F}$ with ends in $N$ satisfying the conditions $\psi_{\rho}=0$. The equality sign is appropriate only when the $E$-function vanishes along $C_{34}$, that is, only when $y_{i}^{\prime}=p_{i}$ at each point of $C_{34}$. But in that case $C_{34}$ would be an extremal of the field and would coincide with $E_{12}$ since $E_{12}$ is the only extremal of the field with ends in $N$ satisfying the conditions $\psi_{p}=0$.

8. An auxiliary theorem. A normal extremal arc $E_{12}$ is said to satisfy the Clebsch condition III' if at each element $\left(x, y, y^{\prime}, \lambda\right)$ on it the inequality

$$
F_{y_{i^{\prime}} y_{k^{\prime}}} \Pi_{i} \Pi_{k}>0
$$

holds for every set $\left(\Pi_{1}, \cdots, \Pi_{n}\right) \neq(0, \cdots, 0)$ which is a solution of the equations $\phi_{\alpha y_{i}} \Pi_{i}=0$. The arc $E_{12}$ satisfies the Mayer condition IV' if there is no point 3 conjugate to 1 on $E_{12}$ between 1 and 2 or at 2 .

In this section we propose to construct $n$ solutions $U_{i k}, V_{i k}$ of equations (4:13) whose determinant $\left|U_{i k}(x)\right|$ is different from zero on $x_{1} x_{2}$ as stated in Theorem 8:1 below. To do this we consider a normal extremal arc $E_{12}$ that is normal on every sub-interval $x_{1} x_{3}$ and satisfies the conditions III', IV' just described. From the condition III' we conclude that $E_{12}$ is non-singular (IX, p. 735).

LEMмA 8:1. There is an interval $x_{1}<x \leqq x_{1}+h$ on which there is no point 3 conjugate to 1 on $E_{12}$.

This lemma is readily proved by the methods used by Bliss to establish the corresponding theorem for the problem of Lagrange (IX, pp. 737-740). Bliss makes the stronger assumption that $E_{12}$ is normal on every sub-interval $x^{\prime} x^{\prime \prime}$, a restriction which is useful if we wish to show that there are no pairs 
of conjugate points whatsoever on $E_{12}$ defined by values $x^{\prime} x^{\prime \prime}$ on an interval $x_{1} \leqq x \leqq x_{1}+h$. It can, however, be replaced by the weaker hypothesis that $E_{12}$ is normal on every sub-interval $x_{1} x_{3}$ if we wish to consider only the points 3 conjugate to 1 on $E_{12}$.

For every pair of solutions $\left(\eta_{i}, \zeta_{i}\right),\left(u_{i}, v_{i}\right)$ of equations $(4: 13)$ it is known that the expression $\eta_{i} v_{i}-u_{i} \zeta_{i}$ is a constant. If this constant is zero, then the two solutions are called conjugate solutions of equations (4:13). A set of $n$ mutually conjugate solutions of equations (4:13) is said to form a conjugate system of solutions.

Consider now the system of solutions $u_{i k}, v_{i k}$ of equations (4:13) defined in Theorem 6:2. One readily verifies that this system forms a conjugate system if the functions $v_{i k}(x)$ are modified so that they satisfy the relation $z_{i}\left(x_{1}\right)$ $\cdot v_{i k}\left(x_{1}\right)=0$. This can be done by adding to the solution $u_{i k}, v_{i k}$ suitable multiples of the solution $\eta_{i} \equiv 0, \zeta_{i}=z_{i}(x)$. Furthermore, since $E_{12}$ satisfies the condition IV' it follows from Theorem 6:2 and Lemma 8:1 that the determinant $\left|u_{i k}(x)\right|$ is different from zero on the interval $x_{1}<x \leqq x_{2}$. When the matrices $\left\|u_{i k}\right\|,\left\|v_{i k}\right\|$ are multiplied on the right by the inverse of the matrix $\left\|u_{i k}\left(x_{2}\right)\right\|$ a new conjugate system $\eta_{i k}, \zeta_{i k}$ is formed which takes values $\delta_{i k}$, $B_{i k}$ at $x=x_{2}$, where $\delta_{i k}$ equals 0 or 1 according as $i \neq k$ or $i=k$, and $B_{i k}=B_{k i}$. It is clear that the determinant $\left|\eta_{i k}(x)\right|$ is also different from zero on the interval $x_{1}<x \leqq x_{2}$. Hence the $n$-parameter family of solutions of equations $(4: 13)$

$$
\eta_{i}=\eta_{i k} a_{k}, \quad \zeta_{i}=\zeta_{i k} a_{k} \quad\left(x_{1} \leqq x \leqq x_{2}\right)
$$

simply covers a region $\mathfrak{F}$ of points $\left(x, \eta_{1}, \cdots, \eta_{n}\right)$ whose $x$-coördinates lie on the interval $x_{1}<x \leqq x_{2}$. Each arc of this family intersects the hyperplane $x=x_{2}$ in points whose $\eta$-coördinates are the parameters $a_{k}$ defining the arc. Furthermore, on the hyperplane $x=x_{2}$ the Hilbert integral $I_{2}{ }^{*}$ for the $x \eta$ problem defined by the family $(8: 1)$ takes the form

$$
I_{2}^{*}=\int 2 \zeta_{i} d \eta_{i}=\int 2 B_{i k} a_{k} d a_{i}=\int d\left(B_{i k} a_{i} a_{k}\right)
$$

and hence is independent of the path. It follows that the family (8:1) defines a field $\mathfrak{F}$ (IX, p. 733), and the following lemma is estabiished:

LEMMA 8:2. If $\eta_{i k}, \zeta_{i k}$ is a conjugate system of solutions taking at $x=x_{2}$ the values $\delta_{i k}, B_{i k}$ just defined, then the determinant $\left|\eta_{i k}(x)\right|$ is different from zero on the interval $x_{1}<x \leqq x_{2}$. Furthermore the $n$-parameter family (8:1) of solutions of the accessory equations defines a Mayer field over a region $\mathfrak{F}$ of points $\left(x, \eta_{1}\right.$, $\left.\cdots, \eta_{n}\right)$ whose $x$-coördinates lie on the interval $x_{1}<x \leqq x_{2}$.

Lemma 8:3. For every extremal $\Gamma_{34}$ for the $x \eta$-problem joining points $(x, \eta)$ 
$=\left(x_{3}, 0\right)$ and $(x, \eta)=\left(x_{2}, a\right)$, with $x_{1} \leqq x_{3}<x_{2}$, the relation

$$
I_{2}\left(\Gamma_{34}\right)-B_{i k} a_{i} a_{k} \geqq 0
$$

holds, where

$$
I_{2}=\int 2 \omega\left(x, \eta, \eta^{\prime}\right) d x
$$

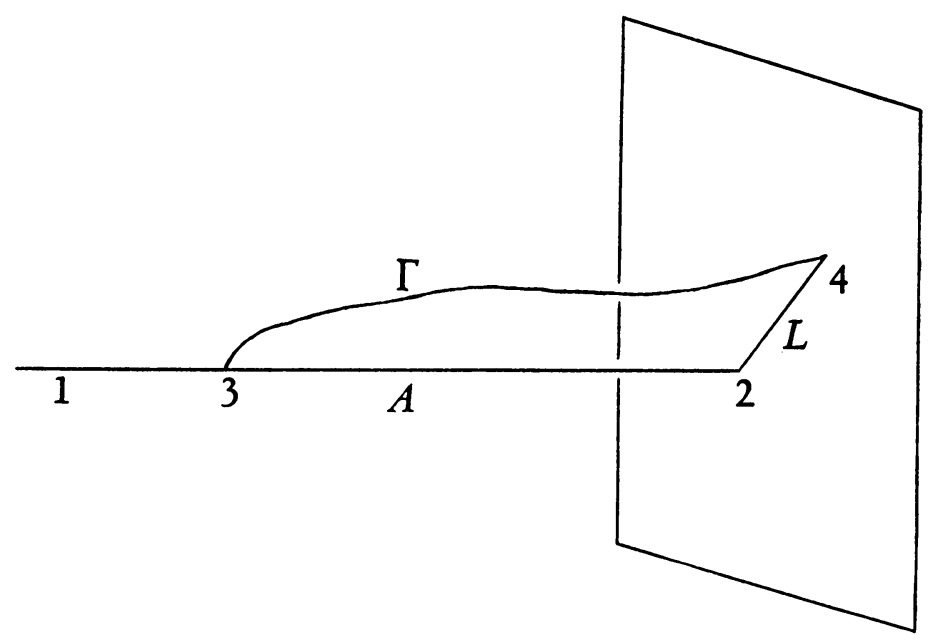

Consider first the case when $x_{3}>x_{1}$. According to Lemma 8:2 the Hilbert integral $I_{2}{ }^{*}$ for the integral $I_{2}$ is independent of the path in $\mathfrak{F}$. Hence

$$
\begin{aligned}
I_{2}{ }^{*}\left(\Gamma_{34}\right) & =I_{2}{ }^{*}\left(A_{32}\right)+I_{2}^{*}\left(L_{24}\right) \\
& =\int_{L_{24}} 2 B_{i k} \eta_{i} d \eta_{k}=B_{i k} a_{i} a_{k} .
\end{aligned}
$$

Since $\Gamma_{34}$ is admissible it follows that

$$
I_{2}\left(\Gamma_{34}\right)-I_{2}{ }^{*}\left(\Gamma_{34}\right)=\int_{\Gamma_{34}} E_{\Omega} d x,
$$

where $E_{\Omega}$ is the Weierstrass $E$-function formed for the function $2 \Omega$. By the use of Taylor's expansion one readily verifies that the condition III' on $E_{12}$ implies that $E_{\Omega} \geqq 0$ along $\Gamma_{34}$. Hence from equations $(8: 3)$ and (8:4) it is clear that the inequality $(8: 2)$ is true whenever $x_{3}>x_{1}$. If now $x_{3}=x_{1}$ then $\Gamma_{34}$ is an extremal of the field and by direct integration it is found that $I_{2}\left(\Gamma_{34}\right)=B_{i k} a_{i} a_{k}$. Hence the lemma is established.

The following theorem gives us the result described at the beginning of this section. 
TheOREM 8:1. Let $U_{i k}, V_{i k}$ be a conjugate system of solutions of equations (4:13) having at $x=x_{2}$ the initial values $\delta_{i k}, H_{i k}=B_{i k}-\delta_{i k}$, where $\delta_{i k}, B_{i k}$ are the values described above. For such a system the determinant $\left|U_{i k}(x)\right|$ is different from zero on the whole interval $x_{1} \leqq x \leqq x_{2}$ and $H_{i k}=H_{k i}$.

In the first place $\left|U_{i k}\left(x_{2}\right)\right|=1$. If now $\left|U_{i k}(x)\right|$ vanishes for a value $x_{3}\left(x_{1} \leqq x_{3}<x_{2}\right)$, then there exist constants $a_{k}$, not all zero, such that $U_{i k}\left(x_{3}\right) a_{k}$ $=0$. The equations

$$
\eta_{i}=U_{i k} a_{k}, \quad \zeta_{i}=V_{i k} a_{k}
$$

define an arc $\Gamma_{34}$ as in Lemma 8:3. By direct integration it is found that for this arc

$$
I_{2}\left(\Gamma_{34}\right)-B_{i k} a_{i} a_{k}=\left(B_{i k}-\delta_{i k}\right) a_{i} a_{k}-B_{i k} a_{i} a_{k}=-a_{i} a_{i}<0 .
$$

This contradicts the result obtained in Lemma $8: 3$. Hence $\left|U_{i k}\left(x_{3}\right)\right|$ is different from zero on the whole interval $x_{1} x_{2}$ as was to be proved.

9. The construction of a field. In order to construct a field we need the following theorem:

THEOREM 9:1. Suppose that an n-parameter family of extremals

$$
y_{i}=y_{i}\left(x, a_{1}, \cdots, a_{n}\right), \quad \lambda_{\alpha}=\lambda_{\alpha}\left(x, a_{1}, \cdots, a_{n}\right)
$$

is intersected by an n-dimensional manifold

$$
x=x_{1}\left(a_{1}, \cdots, a_{n}\right), y_{i}=y_{i}\left[x_{1}\left(a_{1}, \cdots, a_{n}\right), a_{1}, \cdots, a_{n}\right]
$$

and simply covers a region $\mathfrak{F}$ of $x y$-space containing only interior points. If the parameter values of the extremal through the point $(x, y)$ are denoted by $a_{i}(x, y)$, then the region $\mathfrak{F}$ is a field with slope-functions and multipliers

$$
p_{i}(x, y)=y_{i x}[x, a(x, y)], \quad \lambda_{\alpha}(x, y)=\lambda_{\alpha}[x, a(x, y)]
$$

provided that the integral $I^{*}$ is independent of the path on the n-dimensional manifold (9:2).

This theorem has been established by Bliss for the problem of Lagrange (IX, p. 733). The proof is the same for the problem considered here.

THEOREM 9:2. If a normal extremal arc $E_{12}$ is normal on every sub-interval $x_{1} x_{3}$ and satisfies the conditions III', IV', then $E_{12}$ is a member of an $n$-parameter family of extremals (9:1) whose determinant $\left|y_{i a_{k}}\right|$ is different from zero along $E_{12}$. Furthermore $E_{12}$ is an extremal arc of a field $\mathfrak{F}$ simply covered by the family.

To prove this let $W\left(a_{1}, \cdots, a_{n}\right)$ be a function of the form

$$
W(a)=z_{i 2} a_{i}+(1 / 2) H_{i k}\left(a_{i}-y_{i 2}\right)\left(a_{k}-y_{k 2}\right),
$$


where the constants $y_{i 2}, z_{i 2}$ are the values of the functions $y_{i}(x), z_{i}(x)$ defining $E_{12}$ at $x=x_{2}$, and the $H_{i k}$ are the numbers belonging to the conjugate system $U_{i k}, V_{i k}$ defined in Theorem $8: 1$. When in equations (3:6) the set $\left(x_{0}, y_{i 0}\right.$, $\left.z_{i 0}\right)$ is replaced by the set $\left(x_{2}, a_{i}, W_{a_{i}}\right)$, an $n$-parameter family of extremals

$$
\begin{aligned}
& y_{i}=y_{i}\left(x, x_{2}, a, W_{a}\right)=y_{i}(x, a), \\
& z_{i}=z_{i}\left(x, x_{2}, a, W_{a}\right)=z_{i}(x, a)
\end{aligned}
$$

is defined and contains $E_{12}$ for the special values $a_{i}=y_{i 2}$. The multipliers $\lambda_{\alpha}(x, a)$ associated with this family are determined by equations (3:3). Furthermore, since each extremal (9:5) defined by parameter values $a_{i}$ has on it the element $\left(x_{2}, a_{i}, W_{a_{i}}\right)$, it follows that $y_{i a_{k}}=\delta_{i k}, z_{i a_{k}}=W_{a_{i a_{k}}}=H_{i k}$ at $x=x_{2}$. Hence from Theorem 8:1 we conclude that the determinant $\left|y_{i a_{k}}\right|$ is different from zero along each extremal of the family (9:5). This family, therefore, simply covers a neighborhood $\mathfrak{F}$ of $E_{12}$. Moreover, on the hyperplane $x=x_{2}$ the Hilbert integral $I^{*}$ can be expressed in the form

$$
I^{*}=\int F_{y_{i}^{\prime}} d y_{i}=\int W_{a_{i}} d a_{i}=\int d W
$$

and hence is independent of the path. Theorem 9:1 now justifies the theorem that was to be proved.

THEOREM 9:3. Let a normal extremal arc $E_{12}$ be a member of an n-parameter family of extremals $(9: 1)$ whose determinant $\left|y_{i a_{k}}\right|$ is different from zero along $E_{12}$. If the ends of $E_{12}$ satisfy the conditions $\psi_{\rho}=0$, then there is a neighborhood $N$ of these ends in $\left(x_{1} y_{1} x_{2} y_{2}\right)$-space such that $E_{12}$ is the only extremal of the family with ends in $N$ satisfying the conditions $\psi_{\rho}=0$.

To prove this let $E_{12}$ be a member of the family $(9: 1)$ for the special parameter values $\left(x_{10}, x_{20}, a_{0}\right)$. By hypothesis these values satisfy the equations

$$
\psi_{\rho}\left(x_{1}, x_{2}, a\right)=\psi_{\rho}\left[x_{1}, y\left(x_{1}, a\right), x_{2}, y\left(x_{2}, a\right)\right]=0 .
$$

The theorem now follows at once from implicit function theorems if we can show that the matrix

$$
\left\|\psi_{\rho x_{1}}+y_{i 1}^{\prime} \psi_{\rho y_{i 1}} \quad \psi_{\rho x_{2}}+y_{i 2}^{\prime} \psi_{\rho y_{i 2}} \quad \psi_{\rho y_{i 1}} y_{i a_{k}}\left(x_{1}\right)+\psi_{\rho y_{i 2}} y_{i a_{k}}\left(x_{2}\right)\right\|
$$

has rank $n+2$ on $E_{12}$. To do this suppose that it had rank less than $n+2$. Then there would exist constants $b_{1}, b_{2}, c_{k}$, not all zero, such that the relations

$$
\begin{gathered}
\left(\psi_{\rho x_{1}}+y_{i 1}^{\prime} \psi_{\rho y_{i 1}}\right) b_{1}+\left(\psi_{\rho x_{2}}+y_{i 2}^{\prime} \psi_{\rho y_{i 2}}\right) b_{2}+\psi_{\rho y_{i 1}} y_{i a_{k}}\left(x_{1}\right) c_{k}+\psi_{\rho y_{i 2}} y_{i a_{k}}\left(x_{2}\right) c_{k}=0, \\
F_{y^{\prime}}\left(x_{1}\right) y_{i a_{k}}\left(x_{1}\right) c_{k}-F_{y^{\prime}}\left(x_{2}\right) y_{i a_{k}}\left(x_{2}\right) c_{k}=0
\end{gathered}
$$

would hold on $E_{12}$. The last equation is precisely the relation $(2: 3)$ for the 
admissible variations $\eta_{i}=y_{i a_{k}} c_{k}$. On account of the normality of $E_{12}$ the determinant $(2: 5)$ is different from zero on $E_{12}$. Hence we would have

$$
b_{1}=b_{2}=y_{i a_{k}}\left(x_{10}, a_{0}\right) c_{k}=y_{i a_{k}}\left(x_{20}, a_{0}\right) c_{k}=0 \text {. }
$$

But this is impossible since the determinant $\left|y_{i a_{k}}\right|$ is different from zero along $E_{12}$. The matrix $(9: 6)$ therefore has rank $n+2$ on $E_{12}$, and the theorem is established.

10. Sufficient conditions for relative minima. The condition $I$ is defined in $\$ 2$, the Clebsch condition III' and the Mayer condition IV' in $\$ 8$. A normal minimizing arc $E_{12}$ is said to satisfy the Weierstrass condition $\mathrm{II}_{\mathfrak{R}}$ ' if at each element $\left(x, y, y^{\prime}, \lambda\right)$ in a neighborhood $\mathfrak{N}$ of those belonging to $E_{12}$ the inequality

$$
E\left(x, y, y^{\prime}, \lambda, Y^{\prime}\right)>0
$$

holds for every admissible element $\left(x, y, Y^{\prime}\right) \neq\left(x, y, y^{\prime}\right)$.

Theorem 10:1. SUfFicient CONDITIONS FOR A STRONG RELATIVE MINIMUM. Let $E_{12}$ be an admissible arc without corners and with ends satisfying the conditions $\psi_{\rho}=0$. If $E_{12}$ is normal relative to the end conditions $\psi_{\rho}=0$, is normal on every sub-interval $x_{1} x_{3}$ of $x_{1} x_{2}$, and satisfies the conditions $\mathrm{I}, \mathrm{II}_{\mathfrak{N}}$ ', III', IV', then there are neighborhoods $F$ of $E_{12}$ in $x y$-space and $N$ of the ends of $E_{12}$ in $\left(x_{1} y_{1} x_{2} y_{2}\right)$-space such that the inequality $g\left(C_{34}\right)>g\left(E_{12}\right)$ holds for every admissible arc $C_{34}$ in $\mathfrak{F}$ with ends in $N$ satisfying the conditions $\psi_{\rho}=0$ and not identical with $E_{12}$.

To prove this theorem we first notice that the condition I and the normality of $E_{12}$ imply a unique set of multipliers $\lambda_{\alpha}(x)$ and constants $c_{i}$ with which $E_{12}$ satisfies equations $(2: 2)$ and for which $l_{0}=1$, as agreed upon in Theorem 2:2. The condition III' implies further that $E_{12}$ is non-singular and hence must be a single extremal arc, since it has no corners (IX, p. 735). According to Theorem 9:2 we now see that $E_{12}$ is an extremal of a field $\mathfrak{F}$ with slope functions and multipliers $p_{i}(x, y), \lambda_{\alpha}(x, y)$. It follows that if the field $\mathfrak{F}$ is taken sufficiently small, the values $x, y, p_{i}(x ; y), \lambda_{\alpha}(x, y)$ belonging to it will lie in so small a neighborhood of the sets $\left(x, y, y^{\prime}, \lambda\right)$ belonging to $E_{12}$ that the condition $\mathrm{II}_{\mathfrak{R}}$ ' will imply the inequality

$$
E\left(x, y, p(x, y), \lambda(x, y), y^{\prime}\right)>0
$$

for every admissible set $\left(x, y, y^{\prime}\right) \neq(x, y, p)$ in $\mathfrak{F}$. Theorem 9:3 and the fundamental sufficiency theorem 7:2 now justify the theorem that was to be proved. 
Bliss (IX, pp. 736-37) has shown that if an extremal arc $E_{12}$ satisfies the condition III' and is an extremal of a field $\mathfrak{F}$ with slope functions and multipliers $p_{i}(x, y), \lambda_{\alpha}(x, y)$, then the inequality

$$
E\left[x, y, p(x, y), \lambda(x, y), y^{\prime}\right]>0
$$

holds for every admissible set $\left(x, y, y^{\prime}\right) \neq(x, y, p)$ in a neighborhood $\mathfrak{P}$ of the sets $\left(x, y, y^{\prime}\right)$ on $E_{12}$. Hence by arguments like those in the preceding paragraph the following theorem is justified:

THEOREM 10:2. SUFFICIENT CONDITIONS FOR A WEAK RELATIVE MINIMUM. If an admissible arc $E_{12}$ satisfies all the conditions of the preceding theorem except the condition III', then there are neighborhoods $\mathfrak{P}$ of the sets $\left(x, y, y^{\prime}\right)$ on $E_{12}$ and $N$ of the end values $\left(x_{1}, y_{1}, x_{2}, y_{2}\right)$ of $E_{12}$ such that the inequality $g\left(C_{34}\right)>g\left(E_{12}\right)$ is true for every admissible arc $C_{34}$ whose elements $\left(x, y, y^{\prime}\right)$ are all in $\mathfrak{B}$, whose ends are in $N$ and satisfy the conditions $\psi_{\rho}=0$, and which is not identical with $E_{12}$.

Suppose now that the functions $\psi_{\rho}$ are continuous at every pair of distinct or coincident points in a neighborhood of those belonging to $E_{12}$. Bliss has shown that if the ends of $E_{12}$ are the only pair of distinct or coincident points on $E_{12}$ satisfying the conditions $\psi_{\rho}=0$, then for every neighborhood $N$ of the ends of $E_{12}$ in $\left(x_{1} y_{1} x_{2} y_{2}\right)$-space there is a neighborhood $F$ of $E_{12}$ in $x y$-space such that every pair of points $\left(x_{1}, y_{1}\right),\left(x_{2}, y_{2}\right)$ in $\mathfrak{F}$ satisfying the conditions $\psi_{\rho}=0$ are also in $N$ (XII, p. 267). Hence by suitably restricting the neighborhood $\mathfrak{F}$ of $E_{12}$ in Theorem 10:1 we have the following corollary:

COROLlARY 10:1. Let $E_{12}$ be an admissible arc satisfying the conditions described in Theorem 10:1. If further the ends of $E_{12}$ are the only pair of distinct or coincident points on $E_{12}$ satisfying the conditions $\psi_{\rho}=0$, then there is a neighborhood $\mathfrak{F}$ of $E_{12}$ in $x y$-space such that the inequality $g\left(C_{34}\right)>g\left(E_{12}\right)$ holds for every admissible arc $C_{34}$ in $\mathfrak{F}$ with ends satisfying the conditions $\psi_{\rho}=0$ and not identical with $E_{12}$.

A similar corollary can be stated for weak relative minima.

\section{BIBLIOGRAPHX}

I. Kneser, Lehrbuch der Variationsrechnung, Braunschweig, 1900, pp. 227-261.

II. Egorov, Die hinreichenden Bedingungen des Extremums in der Theorie des Mayerschen Problems, Mathematische Annalen, vol. 62 (1906), pp. 371-380.

III. Bolza, Über den anormalen Fall beim Lagrangeschen und Mayerschen Problem mit gemischten Bedingungen und variablen Endpunkten, Mathematische Annalen, vol. 74 (1913), pp. 430-446.

IV. Goursat, A Course in Mathematical Analysis, translated by Hedrick and Dunkel, vol. 2, Part 2. 
V. Bliss, The problem of Mayer with variable end points, these Transactions, vol. 19 (1918), pp. 305-314.

VI. Larew, Necessary conditions in the problem of Mayer in the calculus of variations, these Transactions, vol. 20 (1919), pp. 1-22.

VII. Larew, The Hilbert integral and Mayer fields for the problem of Mayer in the calculus of variations, these Transactions, vol. 26 (1924), pp. 61-67.

VIII. Kneser, Lehrbuch der Variationsrechnung, 2d edition, Braunschweig, 1925, pp. 240-304.

IX. Bliss, The problem of Lagrange in the calculus of variations, American Journal of Mathematics, vol. 52 (1930), pp. 673-742.

$\mathrm{X}$. Morse and Myers, The problems of Lagrange and Mayer with variable end points, Proceedings of the American Academy of Arts and Sciences, vol. 66 (1931), pp. 235-253.

XI. Morse, Sufficient conditions in the problem of Lagrange with variable end conditions, American Journal of Mathematics, vol. 53 (1931), pp. 517-546.

XII. Bliss, The problem of Bolza in the calculus of variations, Annals of Mathematics, vol. 33 (1932), pp. 261-274.

University of Chicago,

Chicago, Ill. 